\title{
Improved critical values for extreme normalized and studentized residuals in Gauss-Markov models
}

\author{
Rüdiger Lehmann \\ Dresden University of Applied Sciences \\ Faculty of Spatial Information \\ Friedrich-List-Platz 1 \\ D-01069 Dresden, GERMANY \\ Phone +493514623146 \\ Fax $\quad+493514622191$ \\ mailto:r.lehmann@htw-dresden.de
}

\begin{abstract}
We investigate extreme studentized and normalized residuals as test statistics for outlier detection in the Gauss-Markov model possibly not of full rank. We show how critical values (quantile values) of such test statistics are derived from the probability distribution of a single studentized or normalized residual by dividing the level of error probability by the number of residuals. This derivation neglects dependencies between the residuals. We suggest improving this by a procedure based on the Monte Carlo method for the numerical computation of such critical values up to arbitrary precision. Results for free leveling networks reveal significant differences to the values used so far. We also show how to compute those critical values for non-normal error distributions. The results prove that the critical values are very sensitive to the type of error distribution.
\end{abstract}

\section{Keywords}

Outlier detection, Gauss-Markov model, Hypothesis testing

\section{Introduction}

One of today's major challenges of geodetic data analysis is gross error detection. In geodesy a gross error is a measurement deviation that is assumed to be generated by a stochastic process of a significantly different characteristic from what is assumed in the stochastic model of the parameter estimation. Typically the statistical dispersion of this process is much larger. Gross errors are also referred to as outliers in Applied Statistics. There are some more or less tentative definitions of an outlier. We quote one of the most popular definitions given be Hawkins (1980): "An outlier is an observation which deviates so much from the other observations as to arouse suspicions that it was generated by a different mechanism". This is very much equivalent to a gross error in Geodesy. We do not dwell on this terminological issue, but use the term "outlier" throughout the rest of the paper.

The quality of parameter estimation using the classical method of least squares is very much affected by outliers. In the last decades a toolbox of robust estimation methods has evolved. They are able to produce reasonable results even if the observations contain some outliers. We do not go into details, but refer to (Anderson 2008) for details.

In Geodesy we often try to identify outliers in the framework of a statistical hypothesis testing and down-weight or remove them from the input data set. If the number of outliers is small and if our data set is sufficiently redundant then we may not lose a significant amount of information. But we 
retain the advantage that we can employ simple and efficient tools of geodetic data analysis like classical least squares estimation. In the following we exclusively pursue this approach.

The best established method for identification of outliers in geodetic data analysis is data snooping. This method is due to Baarda (1968). It was later extended by Pope (1976) to the case that the accuracy of the observations is a priori unknown. Although data snooping was first introduced for the adjustment of geodetic networks, it is a generally applicable method. First we perform the so-called global test of the model. Here we use the weighted sum of squares of the least squares residuals as a test statistic, i.e. as a value indicating outliers or other inconsistencies in the functional or stochastic model. If this test statistic is smaller than some critical value, i.e. a quantile value of its probability distribution, then we accept the hypothesis that the observations do not contain outliers.

Otherwise, and if other deficiencies of the functional and stochastic model can be ruled out, we have to detect and eliminate one or more outliers. In order to actually find the outlying observation we perform the individual or local test, where the individual residuals are considered. The aim of this paper is to compute improved critical values of the test statistics used here.

Since data snooping is based on a statistical hypothesis testing, it may lead to a false decision as follows:

$$
\begin{array}{lll}
\text { type I error } & \text { rejection of a true hypothesis } & \text { probability level } \alpha \\
\text { type II error } & \text { acceptance of a false hypothesis } & \text { probability level } \beta
\end{array}
$$

The computation of the critical values of the test statistics for this test makes a severe neglect, which is no longer necessary to make, when fast computers are available. We will show how to improve the computation of such critical values.

Lehmann (2010) and Lehmann and Scheffler (2011) pose the problem how to determine the optimal levels of type I error probabilities for global and local tests in data snooping. If these levels are chosen too low then we get too large critical values and many outliers remain undetected. If on the contrary these levels are chosen too high then we get too small critical values and it is likely that good observations are eliminated. How to strike a balance between these impairments of parameter estimation? In the papers quoted above it is shown how to use the Monte Carlo method for this purpose.

A different approach introduces fuzzy sets for outlier detection (Aliosmanoglu and Akyilmaz 2001, Wieser 2001).The set of outliers is considered as a fuzzy set and the membership function is derived from the difference between test statistic and critical value. Also here improved critical values of the test statistic would be needed.

The outline of the paper is as follows: First we derive extreme normalized and studentized residuals as test statistics for outliers in the Gauss-Markov model possibly not of full rank. Then we show how to derive critical values by means of the Monte Carlo method. Next we study three standard geodetic Gauss-Markov models. It will turn out that there are significant differences between the classical critical values and the related quantities computed by the Monte Carlo method. 


\section{Gauss-Markov model not of full rank}

To relate the parameters to the observations, we begin with the familiar Gauss-Markov model in linearized form:

$$
y=A \xi+e, \quad E\{e\}=0, \quad D\{e\}=\sigma_{0}^{2} P^{-1}
$$

$y$ is the $n \times 1$-vector of known observations, $\xi$ is the $u \times 1$-vector of unknown parameters, $A$ is the $n \times u$ matrix relating observations and parameters. $e$ is the $n \times 1$-vector of unknown observations errors. $E$ and $D$ denote the operators of expectation and dispersion. $P$ is the known $n \times n$-matrix of weights. $\sigma_{0}^{2}$ is a variance factor, which may or may not be known. $e$ is a vector of random variates and so is $y$.

For the sake of universal applicability the system may or may not be of full rank:

$$
\operatorname{rank}(A)=q \leq u
$$

The least squares solution for the estimated parameters reads

$$
\hat{\xi}=\left(A^{T} P A\right)^{-} A^{T} P y
$$

with superscript "-" denoting some generalized inverse matrix. If $q=u$, this solution is also a best linear unbiased estimate, otherwise $\hat{\xi}$ may not be unique. The residuals (estimated observation errors) are always unique and we obtain them by

$$
\begin{gathered}
\hat{e}=y-A \hat{\xi} \\
=y-A\left(A^{T} P A\right)^{-} A^{T} P y \\
=A \xi+e-A\left(A^{T} P A\right)^{-} A^{T} P(A \xi+e) \\
=e-A\left(A^{T} P A\right)^{-} A^{T} P e=R e
\end{gathered}
$$

This rewriting invokes a property of generalized inverses (cf. Koch 1999). $R$ is known as the redundancy matrix. The dispersion of the residuals reads

$$
D\{\hat{e}\}=\sigma_{0}^{2}\left(P^{-1}-A\left(A^{T} P A\right)^{-} A^{T}\right)=\sigma_{0}^{2} R P^{-1}=\sigma_{0}^{2} Q_{\hat{e} \hat{e}}
$$

where $Q_{\hat{e} \hat{e}}$ denotes the cofactor matrix of the residuals. Therefrom we may derive an unbiased estimate of the variance factor $\sigma_{0}^{2}$ as

$$
\hat{\sigma}_{0}^{2}=\frac{\hat{e}^{T} P \hat{e}}{n-q}
$$

All estimates $\hat{\xi}, \hat{e}, \hat{\sigma}_{0}^{2}$ are functions of $e$ and consequently random variates or random vectors.

\section{$3 \quad$ Normalized and studentized residuals as test statistics for outliers}

For hypothesis testing it is important to assume a distribution of the observation errors $e$ not being outliers. This is typically a central Gaussian distribution:

$$
e \sim N\left(0, \sigma_{0}^{2} P^{-1}\right)
$$


Under this assumption the least squares solution (2) is also a maximum likelihood solution (Koch 1999).

A test statistic is a quantity which assumes extreme values in the case that a certain null hypothesis $H_{0}$ is not fulfilled. In the case of outlier detection this null hypothesis is:

$$
H_{0} \text { : No observation } y_{\mathrm{i}}, i=1, \ldots, n \text { is affected by outliers. }
$$

A possible alternative hypothesis is

$$
H_{A}{ }^{(i)} \text { : The observation } y_{i} \text { for some fixed } i \text { is an outlier. }
$$

According to the pioneering work of Baarda (1968), the decision can be based on the value of the normalized residual

$$
w_{i, \mathrm{norm}}=\frac{\hat{e}_{i}}{\sigma_{0} \sqrt{q_{e_{i} e_{i}}}}
$$

as a test statistic, where $q_{e_{i} e_{i}}$ denotes the $i$-th diagonal element of $Q_{\hat{e} \hat{e}}$ in (4). $w_{i, \text { norm }}$ is by definition (7) a random variate. If $H_{0}$ holds true then $w_{i, \text { norm }} \sim N(0,1)$ is easily derived. For example individual values of $\left|w_{i, \text { norm }}\right|>1.96$ occur only with a probability of $\alpha=0.05$. If we find $\left|w_{i, \text { norm }}\right|>1.96$ for some $y_{i}$, we may reject $H_{0}$. This induces a probability of type I decision error (rejecting $H_{0}$ if it is true) of $\alpha=0.05$. Other critical values are 1.64 for $\alpha=0.10$ and 2.58 for $\alpha=0.01$. In general the critical value reads

$$
c_{\text {norm }}=\Phi^{-1}\left(1-\frac{\alpha}{2}\right)
$$

where $\Phi$ denotes the cumulative distribution function (cdf) of $N(0,1)$. Note that we perform a twosided test of the form $\left|w_{i, \text { norm }}\right| \leq c_{\text {norm. }}$. That is why we have $\alpha / 2$ in (8).

If $\sigma_{0}$ is not known, we may use a test statistic of the form

$$
w_{i, \text { stud }}=\frac{\hat{e}_{i}}{\hat{\sigma}_{0} \sqrt{q_{e_{i} e_{i}}}}
$$

suggested by Pope (1976). It is known as studentized residual. $w_{i, \text { stud }}$ is by definition (9) a random variate. If $H_{0}$ holds true, it has been shown by Pope (1976) that $w_{i, \text { stud }} \sim \tau(1, n-q-1)$. This distribution is known as $\tau$-distribution with 1 and $n$ - $q$ - 1 degrees of freedom.

Quantiles of the $\tau$-distribution can be computed from quantiles of Fishers $F$-distribution, or in our case where the first degree of freedom is 1 , also from Student's- $t$ distribution. In general, the critical value for (9) can be computed as

$$
c_{\text {stud }}=\sqrt{\frac{(n-q) t_{n-q-1}^{2}(\alpha / 2)}{n-q-1+t_{n-q-1}^{2}(\alpha / 2)}}
$$

where $t$ denotes the quantile of the Student's- $t$ distribution with $n-q-1$ degrees of freedom (Pope 1976). Note that we perform a two-sided test of the form $\left|w_{i, \text { stud }}\right| \leq c_{\text {stud. }}$. That is why we have $\alpha / 2$ in (10). From (10) we deduce 
$0 \leq c_{\text {stud }}<\sqrt{n-q}$

\section{$4 \quad$ Testing against $\boldsymbol{n}$ alternative hypotheses}

Usually it is not known which observation $y_{i}$ may be an outlier. Therefore a more appropriate alternative hypothesis would be

$H_{A}$ : There is at least one outlier in the vector of observations $y$.

Since $H_{A}=H_{\mathrm{A}}{ }^{(1)} \vee H_{\mathrm{A}}{ }^{(2)} \vee \ldots \vee H_{\mathrm{A}}{ }^{(\mathrm{n})}$ where each $H_{\mathrm{A}}{ }^{(\mathrm{i})}$ denotes an alternative hypothesis from the preceding section, this is equivalent to testing $H_{0}$ against a sequence of alternative hypotheses

$$
H_{A}^{(1)}, \ldots, H_{A}^{(n)}
$$

Let $w_{i}, i=1, \ldots, n$ denote either normalized or studentized residuals from (7) or (9). $H_{0}$ is rejected if $\left|w_{i}\right|$ exceeds a critical value $c$ for any $i=1, \ldots, n$. Otherwise it is accepted. Therefore the test statistic coming into effect is

$$
w=\max _{\mathrm{i}=1, \ldots, \mathrm{n}}\left|w_{i}\right|
$$

A true $H_{0}$ is rejected after $n$ tests if it is rejected in any of the $n$ tests. The probability that a true $H_{0}$ is accepted in test $i$ is $1-\alpha, i=1, \ldots, n$. If there is an outlier in any observation and since $R$ in (3) is not a diagonal matrix, it is likely that more than one residual is large. Therefore $H_{0}$ is likely to be rejected in more than one test, i.e. the test results depend on each other to some degree. For the sake of simplicity those dependencies are usually neglected. Then the probability that a true $H_{0}$ is accepted in each test is approximately $(1-\alpha)^{n}$. Hence the probability $\alpha$ ' that a true $H_{0}$ is rejected in any test would be

$$
\alpha^{\prime}=1-(1-\alpha)^{n}
$$

Since $\alpha$ is small, we get $\alpha \approx n \alpha$. This is accounted for by computing the critical value $c$ either by

$$
c_{\text {norm }}=\Phi^{-1}\left(1-\frac{\alpha^{\prime}}{2 n}\right)
$$

or by

$$
c_{\text {stud }}=\sqrt{\frac{(n-q) t_{n-q-1}^{2}\left(\alpha^{\prime} /(2 n)\right)}{n-q-1+t_{n-q-1}^{2}\left(\alpha^{\prime} /(2 n)\right)}}
$$

if $\alpha^{\prime}$ is the desired probability of type I decision error with respect to $H_{A}=H_{\mathrm{A}}{ }^{(1)} \vee H_{\mathrm{A}}{ }^{(2)} \vee \ldots \vee H_{\mathrm{A}}{ }^{(\mathrm{n})}$. This procedure is well known and was suggested by Stefansky (1972) for the first time.

\section{$5 \quad$ Extreme normalized and studentized residuals as test statistics}

As an alternative procedure one could treat the extreme (i.e. maximum absolute) normalized or studentized residuals $w$ in (12) directly as a test statistic. One advantage is that we do not need to neglect dependencies in the hypotheses as in (13). However the distributions of the test statistic cannot be derived from well-known test distributions like normal or $t$-distribution anymore.

Therefore critical values cannot be taken from a statistical table but must be computed numerically. 
If $F_{w}$ denotes the cdf of the test statistic $w$ and $\alpha$ is a given probability of type I decision error, we get the desired critical value $c$ by

$$
c=F_{w}^{-1}(1-\alpha)
$$

Note that using (12) as a test statistic we perform a one-sided test of the form $w \leq c$.

Since $w=w(e)$ is a function of a random vector $e$ (observation errors), its distribution is propagated from (6). Let $E^{n}$ denote the $n$-dimensional Euclidian space of observation errors $e$ and let $A_{c} \subset E^{n}$ denote the subset of acceptance of $H_{0}$, i.e.

$$
A_{c}:=\left\{e \in E^{n}: w(e)<c\right\}
$$

The probability of $w(e)<c$ is the same as of $e \in A_{c}$. Therefore values of the $\operatorname{cdf} F_{w}$ can be computed by

$$
1-\alpha=F_{w}(c)=\int_{A_{c}} \varphi_{e}(e) d e
$$

where $\varphi_{\mathrm{e}}$ denotes the probability density function (pdf) of $e$. In the case of (6) this reads

$$
F_{W}(c)=\frac{\operatorname{det}(P)^{1 / 2}}{(2 \pi)^{n / 2} \sigma_{0}^{n}} \int_{A_{c}} \exp \left(-\frac{e^{T} P e}{2 \sigma_{0}^{2}}\right) d e
$$

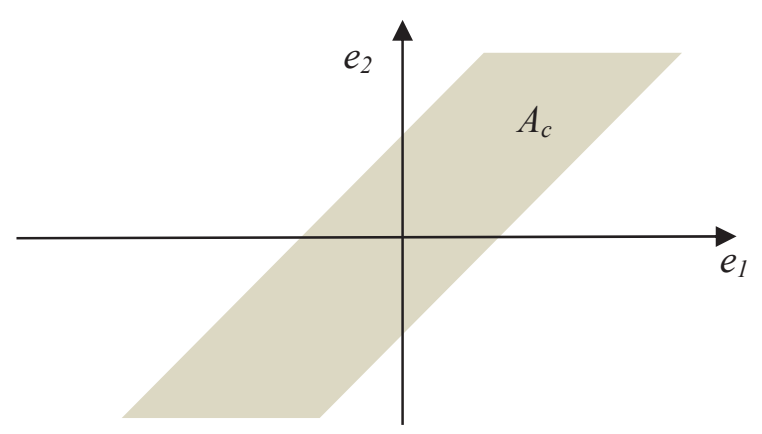

Fig. 1: Set of acceptance Ac in the simplest case of two independent repeated observations $y 1=x+e 1, y 2=x+e 2$ of identical variance, i.e. $A=\left(\begin{array}{ll}1 & 1\end{array}\right) T$ and $P=I$.

Let $I_{c}(e)$ denote the indicator function of $A_{c}$ (i.e. assuming values 1 for $e \in A_{c}$ and 0 otherwise). We get

$$
F_{w}(c)=\int_{E^{n}} I_{c}(e) \cdot \varphi_{e}(e) d e=E\left\{I_{c}\right\}
$$

Since $I_{\mathrm{c}}$ is no simple function, the integral in (19) cannot be evaluated analytically.

\section{Monte Carlo approach}

Monte Carlo methods are able to compute statistical quantities numerically. They are used whenever the functional relationships are analytically not tractable, as is the case for data snooping. The basic idea is to approximate probability distributions by frequency distributions of computer random 
experiments performed using pseudo random numbers. The convergence is only of the order of $m^{-1 / 2}$ if $m$ is the number of experiments performed, but unlike other methods, this order does not depend on the dimension of the data space (Tanizaki 2004), i.e. the number of observations $n$ in Geodesy, which is typically very large.

Since the advent of fast computers we use Monte Carlo methods in Geodesy (e.g. Lehmann 1994, Alkhatib et al. 2009). Those methods have already been applied in outlier detection (e.g. Koch 2007, Lehmann and Scheffler 2011).

In essence the expectation in (19) is approximated by the arithmetic mean

$$
F_{w}(c)=E\left\{I_{c}\right\} \approx \operatorname{mean}\left\{I_{c}\right\}=\frac{1}{m} \sum_{k=1}^{N} I_{c}\left(e_{k}\right)
$$

computed by pseudo random vectors $e_{k}, k=1, \ldots, m$ of the desired distribution.

According to (6) we chose the multivariate normal distribution for $e$. We apply the Box-Muller method (Box and Muller 1958), a common and efficient method for generation of normal pseudo random numbers. It needs uniform pseudo random numbers in the interval $[0,1)$ coming from a modern Mersenne twister generator with period $2^{19937}-1$.

\section{$7 \quad$ Computation procedure}

Firstly note that the distributions of normalized or studentized residuals are independent of $\sigma_{0}$. Consequently the same holds true for $w$ in (12). If we compute a pseudo random vector for $e$ and we do not know $\sigma_{0}$, we may use any positive value instead. The value will be cancelled in (7) or (9).

Secondly normalized or studentized residuals depend on the observation errors $e$, but not on the true values $A \xi$ of the observed quantities. This has been shown in (3).

Thirdly the joint distribution of the vector of normalized or studentized residuals depends on the elements of $Q_{\hat{e} \hat{e}}$. Only the effect of the main diagonal elements $q_{e_{i} e_{i}}$ is cancelled by the denominator of (7) or (9). If we compute the absolute maximum $w$ in (12), we retain the effect of the off-diagonal elements of $Q_{\hat{e} \hat{e}}$. Therefore the distribution of $w$ will in general depend on those elements and in this way on the matrices $A$ and $P$, see (3), (4).

The following procedure yields arbitrarily precise approximations of the desired critical values of $w$ in (12):

1. Compute a sequence of $m$ pseudo random vectors $e_{k}, k=1, \ldots, m$ of the desired distribution, e.g. from (6). $m$ is known as the number of Monte Carlo experiments.

2. For each $e_{k}, k=1, \ldots, m$ estimate residuals $\hat{e}_{k}$ by (3) and compute the test statistic $w_{k}$ by (7) or (9) and (12). The frequency distribution of $w_{k}$ is an approximation of the probability distribution of $w$.

3. For some critical value $c$ we would get $I_{c}\left(e_{\mathrm{k}}\right)=1$ for $w_{k}<c$ and $I_{c}\left(e_{\mathrm{k}}\right)=0$ otherwise. Hence, the desired mean $\left\{I_{c}\right\}$ is the fraction of $w_{k}$ smaller than $c . c$ must be determined such that this fraction becomes 1- $\alpha$. For this purpose, sort vector $w_{k}$ numerically, getting a sorted vector $w_{k}^{\prime}$ such that $w_{1}^{\prime} \leq w_{2}^{\prime} \leq \cdots \leq w_{m}^{\prime}$. Determine the critical value 


$$
c=\frac{1}{2}\left(w_{[(1-\alpha) m]}^{\prime}+w_{[(1-\alpha) m]+1}^{\prime}\right)
$$

where $[\bullet]$ denotes rounding down to the next integer. Note that this can be done for a sequence of values $\alpha$ in parallel.

4. On order to ensure that the approximate values (21) are close to the true values $F_{\mathrm{w}}{ }^{-1}(1-\alpha)$, one should observe the convergence of the procedure as $m \rightarrow \infty$ and implement a suitable termination criterion. We suggest that the procedure should be terminated as soon as $c$ in (21) fluctuates by no more than $1 \%$.

Such a strategy has been used already by Lemeshko and Lemeshko (2005), but only for extreme studentized residuals and only for outlier detection in samples, which is equivalent to the case of repeated observations in Geodesy. In sections 8-10 we will display the results for a less trivial geodetic Gauss-Markov model.

\section{A free leveling network}

Consider a free leveling network with square loops forming a checked pattern (see Fig. 2). Observations are uncorrelated leveled height differences of identical variance $\sigma_{0}^{2}$. We apply the Gauss Markov model (1) with $P=I$ and $A$-matrix of the form

$$
A=\left(\begin{array}{cccccc}
1 & -1 & 0 & \cdots & 0 & 0 \\
1 & 0 & -1 & \cdots & 0 & 0 \\
1 & 0 & 0 & \cdots & 0 & 0 \\
\vdots & \vdots & \vdots & \ddots & \vdots & \vdots \\
0 & 0 & 0 & \cdots & 1 & -1
\end{array}\right)
$$

For a free leveling network we get $\operatorname{rank}(A)=q=u-1$. The right inverse in (2),(3),(4) is computed as usual by the constraint $\Sigma \xi=0$, see (Koch 1999). Note that the actual observations are not required to compute improved critical values (21).

Example: In the case of 2 loops and an "off the reel" ordering of the observations we get

$$
A=\left(\begin{array}{cccccc}
1 & 0 & -1 & 0 & 0 & 0 \\
-1 & 1 & 0 & 0 & 0 & 0 \\
0 & -1 & 0 & 1 & 0 & 0 \\
0 & 0 & 1 & -1 & 0 & 0 \\
0 & 0 & -1 & 0 & 1 & 0 \\
0 & 0 & 0 & 0 & -1 & 1 \\
0 & 0 & 0 & 1 & 0 & -1
\end{array}\right)
$$

The cofactor matrix of the residuals reads

$$
Q_{\hat{e} \hat{e}}=\left(\begin{array}{cccccc}
+0.27 & +0.27 & +0.27 & -0.07 & -0.07 & -0.07 \\
+0.27 & +0.27 & +0.27 & -0.07 & -0.07 & -0.07 \\
+0.27 & +0.27 & +0.27 & -0.07 & -0.07 & -0.07 \\
+0.20 & +0.20 & +0.20 & +0.20 & +0.20 & +0.20 \\
-0.07 & -0.07 & -0.07 & +0.27 & +0.27 & +0.27 \\
-0.07 & -0.07 & -0.07 & +0.27 & +0.27 & +0.27 \\
-0.07 & -0.07 & -0.07 & +0.27 & +0.27 & +0.27
\end{array}\right)
$$




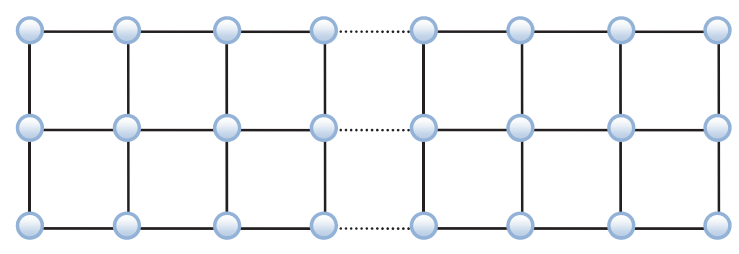

Fig. 2: Leveling network of variable size with square loops forming a checked pattern

First of all, we intend to investigate the convergence of the Monte Carlo procedure described in section 7. Fig. 3 shows how the approximate critical values (21) converge to their true value as $m$ increases. The computation is performed for $2 \times 3$ leveling loops, where $n=15$ and $u=12$. At $m=10000$ we observe only minor changes of $c$ at a relative magnitude of about $1 \%$.

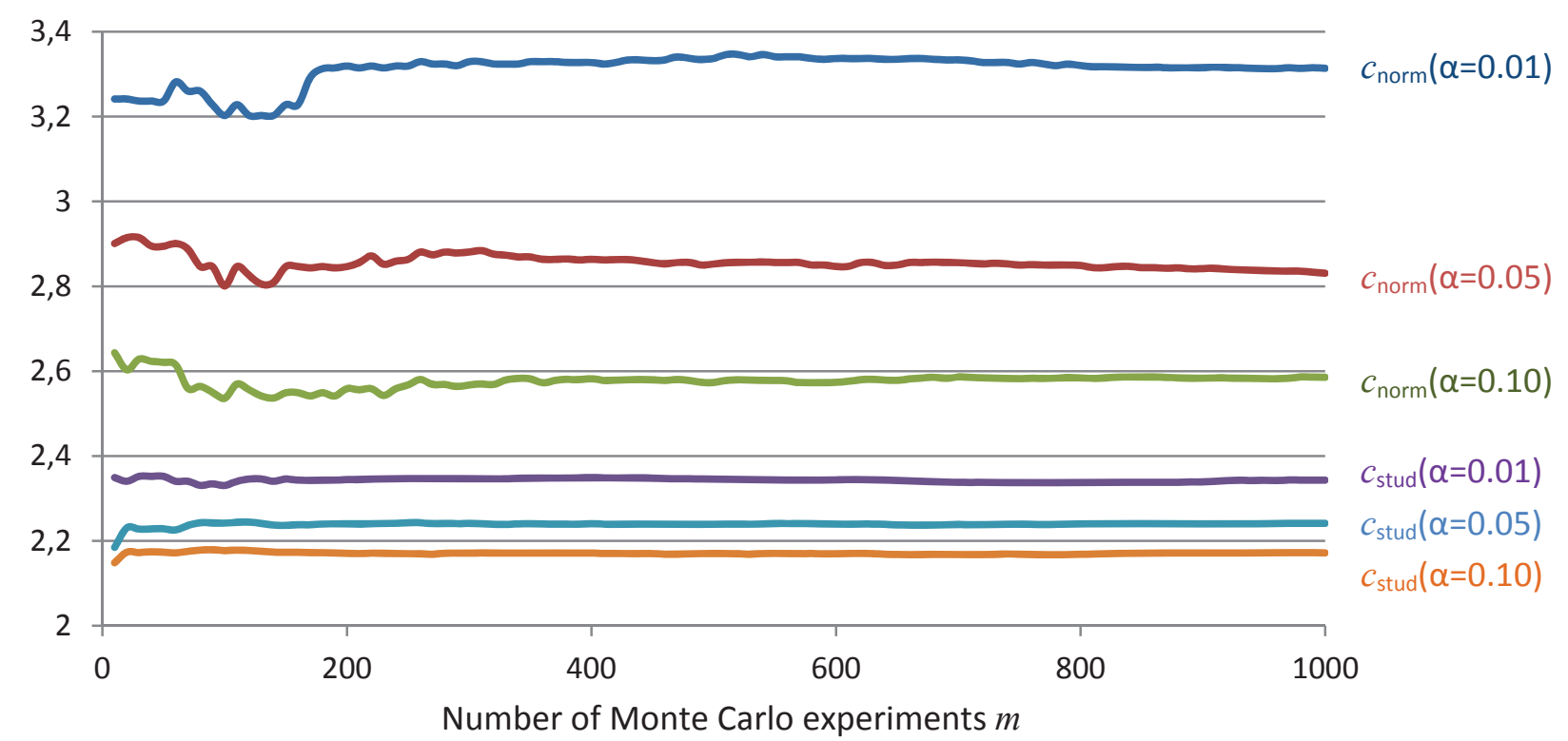

Fig. 3: Critical values (21) of extreme normalized and studentized residuals for $2 \times 3$ leveling loops vs. number of Monte Carlo experiments $m$

To be on the safe side, we decided to double the computational effort to $m=20000$ throughout the following computations.

Next we investigate the critical values of extreme normalized residuals for various sizes of networks starting from $2 \times 1$ loops. Adding two new loops increases the numbers of observations $n$ by 5 and the number of parameters $u$ by 3 . The result is displayed in Fig. 4 . We observe that the improved critical values (21) are always smaller than those values computed by (14) neglecting dependencies between the $n$ tests. In other word, under the condition that $H_{0}$ is true, it is less likely to get large extreme normalized residuals than predicted by (14). For a leveling network this can be understood as follows:

If there is according to (6) a large measurement error then we get large loop misclosures in the adjoining loops and all residuals in these loops tend to be large in magnitude. If aligned in the same sense of direction, the residuals also tend to have the same sign. If all measurement error happen to be small then all residuals tend to be small. Hence the residuals are correlated. In (13) we have 
neglected such correlations. In this case and if one residual is small, we still suppose that the neighboring residual in this loop can be large in magnitude. In this way using (14) we overestimate the probability of large extreme normalized residuals and the dashed curve in Fig. 4 is always above the solid curve. This line of reasoning applies to positive and negative correlations. (In fact, one can change positive into negative correlations and vice versa by changing the sign of one observation.) Therefore the dashed curve is always above the solid curve in Fig. 4. The effect is less pronounced for larger networks because the correlations attenuate with distance and in a large network almost all pairs of observations are spatially separated.

Consequently at the same level of error probability the improved critical values (21) detect more outliers than those computed by (7).

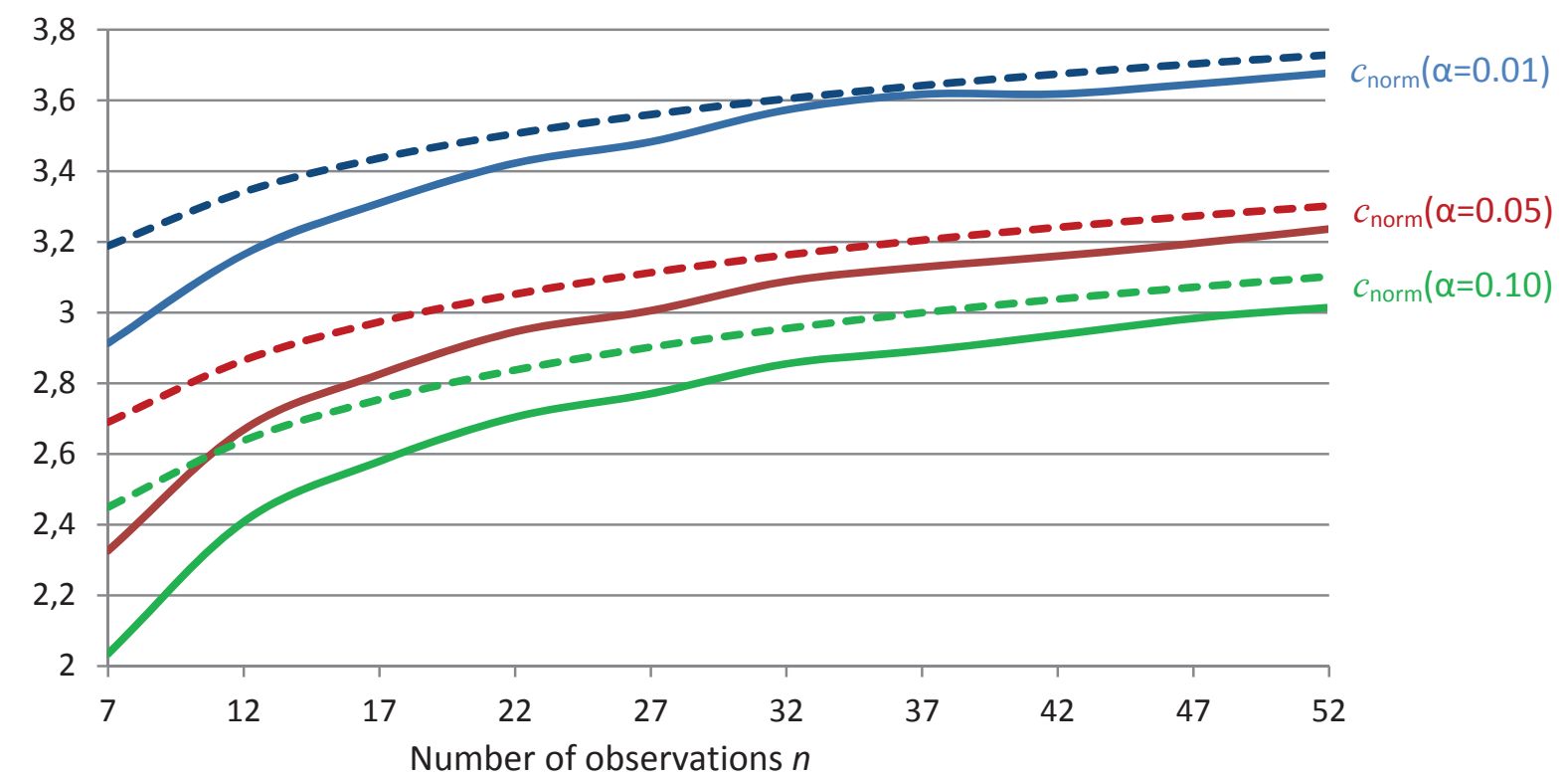

Fig. 4: Critical values of extreme normalized residuals for the free leveling network. Solid curves: obtained from (21) by $m=20000$ Monte Carlo experiments. Dashed curves: obtained from (14)

We repeat this computation for extreme studentized residuals. The result is displayed in Fig. 5. First of all we note that the critical values are smaller than for the extreme normalized residuals. This is comprehensible from definition (9). If a residual $e_{\mathrm{i}}$ is large than it is probable that also $\hat{\sigma}_{0}$ in (5) is large, partly mitigating the effect in (9). Consequently studentized residuals tend to be smaller than normalized residuals. But as the number of observations $n$ increases, this effect more and more vanishes by averaging of the residuals in (5). Indeed we observe in Fig. 4 and 5 that the extreme studentized residuals increase faster than the extreme normalized residuals with the number of observations $n$.

Another difference between Fig. 4 and 5 is that in small networks the critical values of the studentized residuals hardly depend on $\alpha$. The reason is that here a large part of studentized residuals concentrate near the theoretical upper bound of $c$ according to (11), see dotted curve in Fig. 5. Overestimation as before is hardly possible. Differences between the improved critical values (21) and those values computed by (15) come into effect when the network gets larger. Again the values obtained from (14) are larger for the same reason as in the case of normalized residuals. The 
difference is less pronounced here because of weaker correlations in larger networks, cf. discussion above.

Larger differences can be expected for models where the correlations do not diminish with size, e.g. if in a geodetic network also long traverses have been observed.

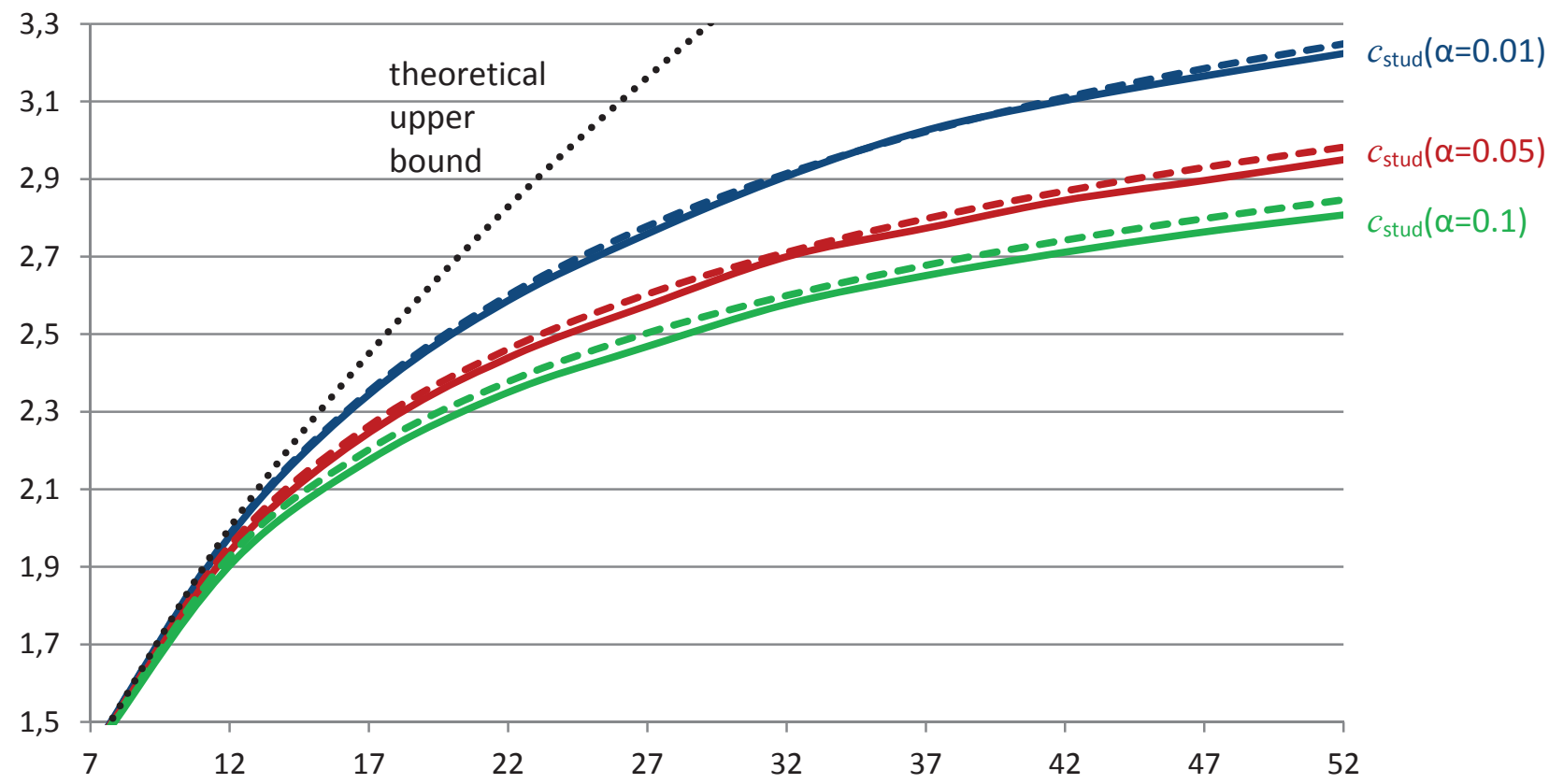

Number of observations $n$

Fig. 5: Critical values of extreme studentized residuals for the free leveling network. Solid curves: obtained from (21) by $m=20000$ Monte Carlo experiments. Dashed curves: obtained from (15). Dotted curve: theoretical upper bound according to (11)

\section{Correlated observations}

All derivations are valid also for correlated observations where $P$ is not a diagonal matrix. The generation of correlated pseudo random numbers $e$ with a desired covariance matrix $D\{e\}=\sigma_{0}^{2} P^{-1}$ in (1) from uncorrelated pseudo random numbers $e$ ' with covariance matrix $I$ can be done by the transform

$$
e=U e^{\prime}
$$

with any $n \times n$-matrix $U$ fulfilling

$$
U U^{T}=D\{e\}
$$

This property is a consequence of covariance propagation.

We repeat the computations of the preceding section with a covariance matrix 
$D\{e\}=\sigma_{0}^{2}\left(\begin{array}{cccccc}1 & \rho & \rho & \cdots & \rho & \rho \\ \rho & 1 & \rho & \cdots & \rho & \rho \\ \rho & \rho & 1 & \cdots & \rho & \rho \\ \vdots & \vdots & \vdots & \vdots & \vdots & \vdots \\ \rho & \rho & \rho & \ddots & 1 & \rho \\ \rho & \rho & \rho & \cdots & \rho & 1\end{array}\right)$

where all pairs of observations are correlated with a correlation coefficient of $0 \leq \rho<1$. This correlation can be assumed to be caused by using the same leveling equipment throughout the whole network. A simple matrix $U$ having property (24) is a $n \times n$-matrix with elements equal to

$$
u_{i j}=\frac{1}{n}(\sqrt{1+(n-1) \rho}+\sqrt{1-\rho})+\delta_{i j} \sqrt{1-\rho}
$$

where $\delta_{i j}$ denotes Kronecker's delta.

Example: For $\rho=0.9$ the cofactor matrix of the residuals (23) is modified to

$$
Q_{\hat{e} \hat{e}}=\left(\begin{array}{cccccc}
+0.29 & +0.29 & +0.29 & -0.04 & -0.04 & -0.04 \\
+0.29 & +0.29 & +0.29 & -0.04 & -0.04 & -0.04 \\
+0.29 & +0.29 & +0.29 & -0.04 & -0.04 & -0.04 \\
+0.25 & +0.25 & +0.25 & +0.25 & +0.25 & +0.25 \\
-0.04 & -0.04 & -0.04 & +0.29 & +0.29 & +0.29 \\
-0.04 & -0.04 & -0.04 & +0.29 & +0.29 & +0.29 \\
-0.04 & -0.04 & -0.04 & +0.29 & +0.29 & +0.29
\end{array}\right)
$$

Although $\rho=0.9$ is a quite large correlation coefficient, (23) and (26) are not very much different. We conclude that correlations of the residuals are much more caused be the functional than by the stochastic relationships of the observations. So it is no surprise that the critical values do not strongly depend on the stochastic correlations introduced by (25). In Tab. 1 and 2 we only display the results for $\alpha=0.05$. Other values of $\alpha$ yield a similar outcome. We can conclude from these tables that in this case the critical values are practically the same for all correlations.

Tab. 1: Critical values of extreme normalized residuals for the free leveling network and correlated observations (25), $\alpha=0.05$

\begin{tabular}{|c|c|c|c|c|c|}
\hline$n$ & $\begin{array}{c}c \text { from } \\
(14)\end{array}$ & $\begin{array}{c}\text { from (21) } \\
\rho=0\end{array}$ & $\begin{array}{c}\text { from (21) } \\
\rho=0.3\end{array}$ & $\begin{array}{c}\text { from (21) } \\
\rho=0.6\end{array}$ & $\begin{array}{c}\text { from (21) } \\
\rho=0.9\end{array}$ \\
\hline 7 & 2.69 & 2.34 & 2.31 & 2.34 & 2.36 \\
\hline 12 & 2.87 & 2.68 & 2.68 & 2.67 & 2.68 \\
\hline 17 & 2.97 & 2.83 & 2.82 & 2.84 & 2.84 \\
\hline 22 & 3.05 & 2.94 & 2.91 & 2.94 & 2.93 \\
\hline 27 & 3.11 & 3.02 & 3.02 & 3.03 & 3.01 \\
\hline 32 & 3.16 & 3.07 & 3.06 & 3.08 & 3.08 \\
\hline 37 & 3.20 & 3.12 & 3.11 & 3.12 & 3.12 \\
\hline 42 & 3.24 & 3.17 & 3.16 & 3.15 & 3.17 \\
\hline 47 & 3.27 & 3.20 & 3.21 & 3.21 & 3.21 \\
\hline 52 & 3.30 & 3.22 & 3.23 & 3.23 & 3.24 \\
\hline
\end{tabular}

Tab. 2: Same as Tab.1, but studentized rather than normalized residuals 


\begin{tabular}{|c|c|c|c|c|c|}
\hline$n$ & $\begin{array}{c}c \text { from } \\
(15)\end{array}$ & $\begin{array}{c}\text { from (21) } \\
\rho=0\end{array}$ & $\begin{array}{c}\text { from (21) } \\
\rho=0.3\end{array}$ & $\begin{array}{c}\text { from (21) } \\
\rho=0.6\end{array}$ & $\begin{array}{c}\text { from (21) } \\
\rho=0.9\end{array}$ \\
\hline 7 & 1.41 & 1.41 & 1.41 & 1.41 & 1.41 \\
\hline 12 & 1.95 & 1.94 & 1.94 & 1.94 & 1.94 \\
\hline 17 & 2.26 & 2.24 & 2.25 & 2.24 & 2.24 \\
\hline 22 & 2.46 & 2.44 & 2.44 & 2.45 & 2.43 \\
\hline 27 & 2.60 & 2.59 & 2.58 & 2.59 & 2.58 \\
\hline 32 & 2.71 & 2.68 & 2.69 & 2.68 & 2.69 \\
\hline 37 & 2.80 & 2.78 & 2.78 & 2.78 & 2.77 \\
\hline 42 & 2.87 & 2.85 & 2.85 & 2.84 & 2.85 \\
\hline 47 & 2.93 & 2.91 & 2.90 & 2.91 & 2.91 \\
\hline 52 & 2.98 & 2.96 & 2.96 & 2.95 & 2.96 \\
\hline
\end{tabular}

\section{Non-normal error distributions}

Formulas (14) and (15) are only valid if observation errors are normally distributed according to (6).

A further advantage of the Monte Carlo method is that we are not restricted to assumption (6). The only requirement is that we must be able to generate pseudo random numbers of the non-normal error distribution. This is ensured for the most common alternatives like symmetric triangular distribution or Laplace distribution. In both cases we get the inverse cdf explicitly. This enables us to apply the inverse transformation method for the generation of pseudo random numbers according to the symmetric triangular and Laplace distribution (Tanizaki 2004 p.116). Although neglected here, a further interesting alternative is the scale contaminated normal distribution (cf. Lehmann 2012).

We repeat the computations of section 8 using (see Fig. 6)

1. the central symmetric triangular distribution and

2. the central Laplace distribution.

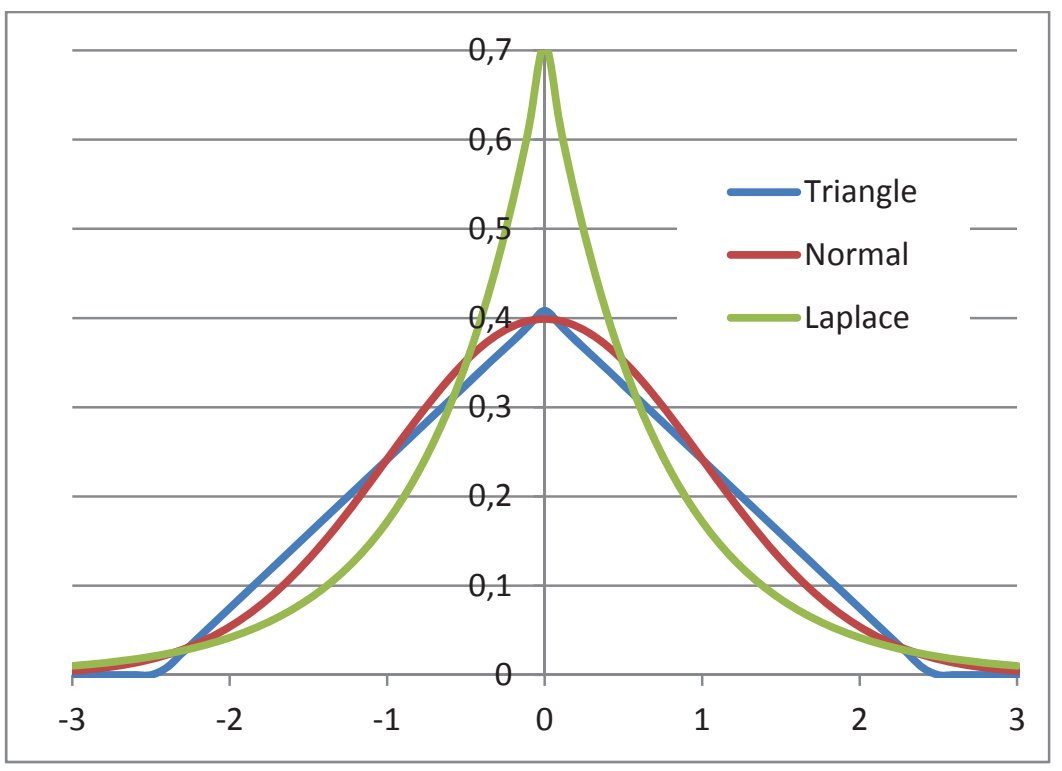

Fig. 6: Probability density functions of distributions used (zero expectation, unit variance) 
Note that again the variance of the pseudo random numbers can be chosen arbitrarily because it cancels in (7) or (9). In Fig. 7 we only display the results for $\alpha=0.05$. Other values of $\alpha$ yield a similar outcome.

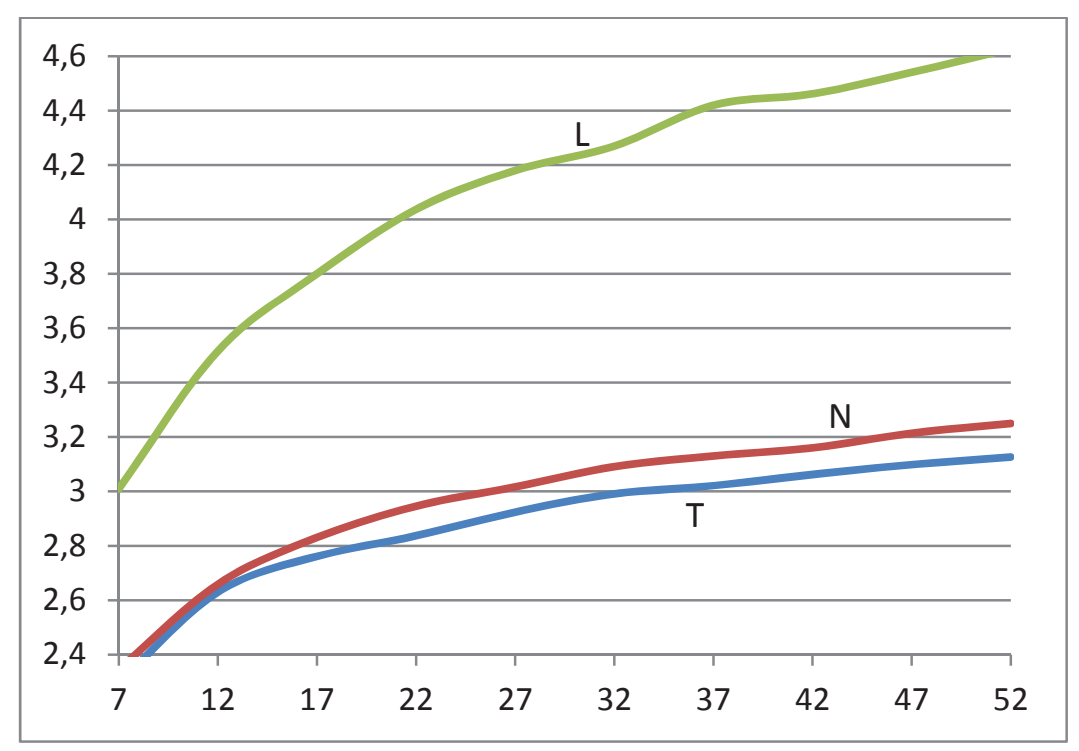

Fig. 7: Critical values of extreme normalized residuals for the free levelling network computed by (21) using the triangular ( $T$ ) distribution (blue), using the normal $(N)$ distribution (red) and using the Laplace (L) distribution (green curve) for $\alpha=0.05$.

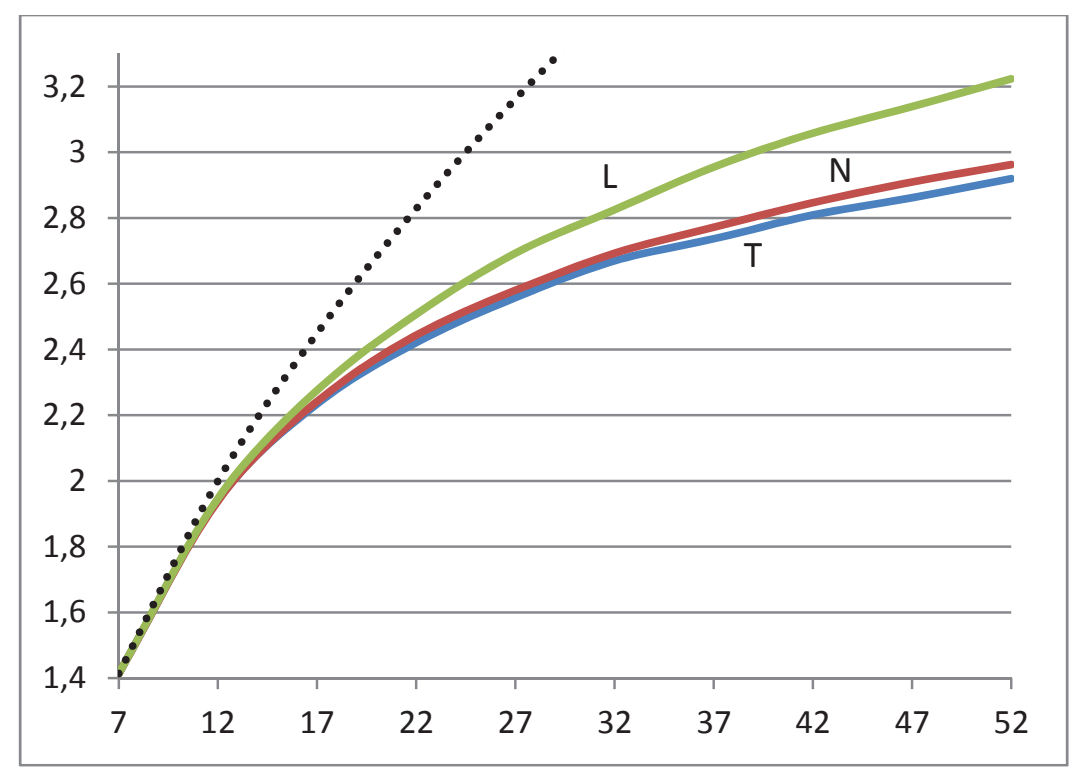

Fig. 8: Same as Fig.7, but studentized rather than normalized residuals. Dotted curve: theoretical upper bound according to (11)

Fig. 7 and 8 show that the critical values depend very much on the type of error distribution, even more than on the functional model. This effect becomes more pronounced as the number of observations increases. The triangular distribution has bounded observation errors. Under the hypothesis $H_{0}$ this makes large extreme normalized or studentized residuals very unlikely. On the contrary, the Laplace distribution has heavy "tails". Here we can expect to get large extreme 
normalized or studentized residuals even if there are no outliers ( $H_{0}$ holds true). As expected, we observe that $c_{T}<c_{N}<c_{\mathrm{L}}$ holds with subscipts as in Fig. 7 and 8.

\section{Considerations regarding the computational workload}

In each Monte Carlo experiment we have to generate $n$ pseudo random numbers $e_{k}$ and evaluate $\hat{e}=R e$. Note that $R$ must be computed only once. In the worst case that $R$ is a dense (not sparse) matrix, we need $2 n^{2}$ floating point operations for $\hat{e}=R e$. The computation of the test statistic has only $O(n)$ operations. Sorting the vector of test statistics typically requires $O(n \log n)$ operations. If $n$ is large, only the evaluation of $\hat{e}=R e$ with dense $R$ deserves consideration.

If we assume a Gauss Markov model with $n=1000$ unknowns and $m=20000$ Monte Carlo experiments, this amounts to $4 \cdot 10^{10}$ floating point operations for only the evaluation of $\hat{e}=R e$. For example on a common Intel Core i7 processor with up to 100 GFLOPS this computation takes less than a second. In general we can expect that minor changes of the functional and stochastic model may not change the improved critical values considerably.

Hence we are not overwhelmed with computational workload. Also remember that (21) can be evaluated efficiently for a sequence of values $\alpha$ in parallel.

\section{Monte Carlo based data snooping}

We make a statement concerning the use of the approach proposed in (Lehmann 2010) and (Lehmann and Scheffler 2011) called "Monte Carlo based data snooping" and show how it relates to the subject of this paper. It finds the optimum level of error probability $\alpha$ as follows: for a number of trial levels $\alpha_{i}, i=1, \ldots, M$ the posterior variance of the estimated parameters is computed and the optimum $\alpha^{*}$,i.e. the value $\alpha_{i}$, for which the posterior variance of the estimated parameters is minimum, is selected and possibly refined by some interpolation. $M$ is a chosen integer large enough as to ensure finding the optimum $\alpha^{*}$. It is suggested to use a geometric progression like $\alpha_{i}=2^{-2-i}$, $\mathrm{i}=1, \ldots, 7$. One can even use the trial critical values $\mathrm{c}_{\mathrm{i}}, i=1, \ldots, M$ instead of error levels finding the optimum $c^{*}$ in the same way. In this case $c_{\mathrm{i}}$ is not computed in any way from $\alpha_{\mathrm{i}}$ by (14) or (15). Therefore Monte Carlo based data snooping does not require the improved critical values derived here. Computation time cannot be saved however, because Monte Carlo based data snooping is also moderately time-consuming.

\section{Conclusions}

The improved critical values promised by the title of this paper cannot exactly be listed in a table as in the classical case, see e.g. (Pope 1976). This would only be possible in special cases, see (Lemeshko and Lemeshko 2005). They depend more or less on the functional and stochastic model. All that can be given is a procedure how to compute the improved values for each model.

The improved critical values (21) are always smaller than the critical values (14),(15) used so far. This means that in the case of no outliers it is less likely that extreme normalized or studentized residuals get very large than predicted by the classical approach of section 4. E.g. if $H_{0}$ is true and is rejected against $H_{A}^{(l)}$ it is more likely that it is rejected also against $H_{A}{ }^{(2)}$ etc. because the test statistics are correlated. This makes the approximation (13) relatively coarse.

Let us consider the case of iterative outlier elimination, i.e. after each detected and eliminated outlier the model is reprocessed and the outlier detection is restarted. This is a standard procedure 
in Geodesy. It is obvious that with the improved critical values we get the same sequence of outliers as in the classical approach of section 4. It only truncates at a later cycle of iteration. In other words, we get the same outliers as with the classical approach and a larger level of error probability $\alpha$. However, it is not easy to derive this larger $\alpha$ somehow from the value $\alpha$ used before.

Finally we suggest applying Monte Carlo methods also in other fields of outlier detection. E.g. there are test statistics for outlier detection, which do not even approximately permit an analytical derivation of a related cdf. Therefore such test statistics have not yet been used in Geodesy. As an example we mention the kurtosis of the normalized residuals (cf. Verma et al. 2008), which is able to detect multiple outliers without the need to a priori specify them. Using the Monte Carlo method critical values for such test statistics can be computed in the same way as for the extreme residuals.

\section{References}

Aliosmanoglu, S.; Akyilmaz, O.: A Comparison between Statistical and Fuzzy Techniques in Outlier Detection. International Association of Geodesy Symposia Symposia, Vol. 125, 382-387, Springer 2001

Alkhatib, H.; Neumann, I.; Kutterer, H.: Uncertainty modeling of random and systematic errors by means of Monte Carlo and fuzzy techniques. Journal of Applied Geodesy, Vol. 3, No. 2, 67-79, 2009

Andersen R: Modern Methods for Robust Regression. Sage Publications London 2008

Baarda, W.: A testing procedure for use in geodetic networks. Netherlands Geodetic Commission, Publication on Geodesy, 2(5), Delft, Netherlands 1968

Box, G. E. P.; Muller M.E.: A Note on the Generation of Random Normal Deviates. The Annals of Mathematical Statistics, Vol. 29, No. 2, 610-611, 1958

Hawkins D.: Identification of Outliers. Chapman and Hall London New York 1980

Koch, K.R.: Parameter Estimation and Hypothesis Testing in Linear Models. Springer Verlag Berlin Heidelberg New York 1999

Koch, K.R.: Outlier Detection in Observations Including Leverage Points by Monte Carlo Simulations. Allgemeine Vermessungsnachrichten. VDE Verlag Berlin Offenbach 2007

Lemeshko, B.Y.; Lemeshko, S.B.: Extending the Application of Grubbs-Type Tests in Rejecting Anomalous Measurements. Measurement Techniques, Vol. 48, No. 6, 536-547, 2005

Lehmann, R.: Adjustment in non-linear models by means of the adaptive Monte-Carlo-Integration (in German). Allgemeine Vermessungsnachrichten Vol. 7/1994, Herbert Wichmann Verlag GmbH Heidelberg 1994

Lehmann, R.: Normalized residuals - how large is too large? (in German). Allgemeine Vermessungsnachrichten Vol. 2/2010, VDE Verlag Berlin Offenbach 2010

Lehmann, R.: Geodetic error calculus by the scale contaminated normal distribution (in German). Allgemeine Vermessungsnachrichten. VDE Verlag Berlin Offenbach 2012 (in print) Lehmann, R.; Scheffler, T.: Monte Carlo based data snooping with application to a geodetic network. Journal of Applied Geodesy, Vol. 5, Issue 3-4, 123-134, 2011

Pope, A.J.: The statistics of residuals and the detection of outliers. NOAA Technical Report NOS65 NGS1, US Department of Commerce, National Geodetic Survey Rockville, Maryland 1976 
Stefansky, W: Rejecting outliers in factorial designs. Technometrics, Vol. 14, 469-479, 1972.

Tanizaki, H.: Computational Methods in Statistics and Econometrics. Marcel Dekker New York, 2004

Verma, SP; Quiroz-Ruiz, A; Díaz-González, L: Critical values for 33 discordancy test variants for outliers in normal samples up to sizes 1000, and applications in quality control in Earth Sciences. Revista Mexicana de Ciencias Geológicas Vol. 25, No. 1, 2008, 82-96

Wieser, A.: Robust and fuzzy techniques for parameter estimation and quality assessment in GPS. Ph.D. dissertation, Graz University of Technology, Austria, 2001 